\title{
Bioinformatics analysis of gene expression alterations conferring drug resistance in tumor samples from melanoma patients with EGFR-activating $B R A F$ mutations
}

\author{
YANG YU* ${ }^{*}$ XUEER WANG* ${ }^{*}$ QINGLIN LI, MIN ZHANG, PENGCHENG XU, \\ YINGHUA CHEN, YUAN YAN and LIN ZHANG \\ Department of Histology and Embryology, School of Basic Medical Sciences, Southern \\ Medical University, Guangzhou, Guangdong 510515, P.R. China \\ Received February 22, 2016; Accepted July 5, 2017
}

DOI: $10.3892 / \mathrm{ol} .2017 .7330$

\begin{abstract}
Melanoma is a highly malignant tumor of the skin melanocytes. Patients with this cancer have a high frequency $(\sim 50 \%)$ of oncogenic BRAF mutations, particularly BRAF V600E. Treatments for melanoma often target $B R A F$ mutations or involve mitogen-activated protein kinase kinase/extracellular signal-regulated kinase inhibitors. A major challenge in melanoma treatment is resistance to $B R A F$ inhibitor treatment, which may be enhanced by the $B R A F$ mutation itself and/or epidermal growth factor receptor (EGFR) activation, leading to poor prognosis. However, no effective clinical treatment exists for patients with EGFR-activating feedback. The aim of the present study was to analyze gene expression changes in tumors from patients with EGFR-activating $B R A F$ mutations during development of drug resistance. RNA-seq data was downloaded from the Gene Expression Omnibus (GEO) database for pre- and post-treatment tumor samples from three melanoma patients with EGFR-activating BRAF V600E mutations, and from The Cancer Genome Atlas (TCGA) melanoma database for tumor and non-tumor samples from patients with the BRAF V600E mutation and unknown EGFR activation status. Using functional enrichment and KEGG pathway analyses, the present study analyzed differentially expressed genes (DEGs) between pre- vs. post-treatment data from the GEO database and tumor or non-tumor sample data from the TCGA database. The results of the present study indicated that functional and structural changes to the plasma membrane
\end{abstract}

Correspondence to: Professor Lin Zhang, Department of Histology and Embryology, School of Basic Medical Sciences, Southern Medical University, 1838 Guangzhou Avenue North, Guangzhou, Guangdong 510515, P.R. China

E-mail: zlilyzh@126.com

${ }^{*}$ Contributed equally

Key words: BRAF V600, melanoma, molecular profiling, treatment resistance may be associated with drug resistance. The present study identified 9 DEGs that were significantly different between tumor and non-tumor samples and also between prior to and following treatment. Thus, it was confirmed that patients with EGFR-activating BRAF V600E mutations undergo gene expression changes during disease development, and during therapy. These findings may provide potential directions for melanoma-specific therapy.

\section{Introduction}

Melanoma is a highly malignant tumor of the skin melanocytes that is insensitive to chemotherapy or radiotherapy (1). The majority of patients with melanoma exhibit a poor prognosis, and the disease is associated with a high mortality rate (2). Patients with melanoma frequently exhibit activation of the BRAF gene due to somatic mutations, with up to $50 \%$ of patients exhibiting $B R A F$ oncogenic mutations $(3,4)$. The most common $B R A F$ mutation in melanoma, BRAF V600E, accounts for $\sim 79 \%$ of $B R A F$ mutations (5). BRAF is a member of the RAF kinase family, which includes ARAF, BRAF and CRAF (6). BRAF mutations can lead to the constitutive activation of downstream signaling through mitogen-activated protein kinase (MAPK) pathways, including the mitogen-activated protein kinase kinase (MEK)-extracellular signal-regulated kinase (ERK) pathway, which subsequently upregulates cell migration and proliferation (7).

Good clinical outcomes have been obtained with melanoma treatments that target $B R A F$ mutants and with MEK/ERK inhibitors. As a first-line clinical treatment for melanoma, vemurafenib (PLX4032) is a potent inhibitor of mutated $B R A F$ and a specific therapy for advanced melanoma (8). However, targeted inhibitors typically only maintain their efficacy for 8-9 months before the tumor develops resistance to the inhibitor, allowing rapid growth to continue (9). Thus, controlling drug resistance is a key issue in melanoma treatment.

A number of studies have attempted to elucidate the mechanisms of drug resistance in melanoma patients. Hepatocyte growth factor (HGF) expression has been observed in the stromal cells of patients carrying $B R A F$ mutations, and an association has been demonstrated between HGF-secreting stromal 
cells and the resistance to Raf inhibitors (10). Another potential cause for the development of resistance is mitogen-activated protein kinase kinase kinase 8 overexpression by cells (11). Mutations of the asparaginyl-tRNA synthetase (NARS) gene were identified in vemurafenib-resistant cells in vitro, and in the lymph node cells of vemurafenib-resistant patients. NARS mutations may be active in the MAPK pathway, leading to the resistance of melanoma cells to targeted inhibitors (12).

The BRAF mutation itself may lead to resistance development. In previous reports, patients with the BRAF V600E mutation exhibited a poor prognosis due to acquired resistance to vemurafenib and trametinib $(13,14)$. Other studies have demonstrated that the BRAF V600E mutation or MEK inhibitor resistance may be associated with epidermal growth factor receptor (EGFR) activation in tumor cells $(15,16)$. For example, Prahallad et al identified that in a subset of patients, BRAF V600E inhibitors may lead to EGFR activation, which, in turn, may enhance the resistance of cancer cells to BRAF inhibitors (15). Sun et al demonstrated that EGFR expression enhances the proliferation of melanoma cells in the presence of inhibitors against BRAF or MEK (16).

There remains no effective clinical treatment for patients with EGFR-activating feedback. Furthermore, it has yet to be established whether patients with EGFR-activating BRAF V600E mutations experience alterations to gene expression prior to and following disease or treatment. Therefore, the aim of the present study was to analyze changes in the expression of genes by melanoma tumors in patients with EGFR-activating $B R A F$ mutations, including during the development of drug resistance. The overall goal was to identify potential drug targets for melanoma treatment-resistant patients.

\section{Materials and methods}

Identification of differentially expressed genes (DEGs) from a public database. All cases were pathologically diagnoses to be skin melanoma while the controls were well identified as have drug resistance with EGFR-activating BRAF mutations. Sample numbers SRR961663, SRR961664, SRR961665, SRR961666, SRR961667 and SRR961668 were downloaded from dataset GSE50535 of the Gene Expression Omnibus (GEO; https://www.ncbi.nlm.nih.gov/geo/). TopHat 2.1.1 and Cufflinks 2.2.1 software packages were utilized to analyze assembly data and differences in the gene expression profiles, respectively (17). RNA-seq data of 130 samples from tumor and non-tumor sites of patients with BRAF V600E mutations of unknown EGFR activation status were downloaded from the Cancer Genome Atlas (TCGA) cutaneous melanoma database (https://cancergenome.nih.gov/). Using the edgeR3.5 package in Bioconductor, DEGs between pre- and post-treatment data from the GEO dataset and between pre-treatment data from the GEO dataset vs. tumor or non-tumor sample data from the TCGA site were analyzed (18). $\mathrm{P}<0.05$ was considered to indicate a statistically significant difference.

Functional enrichment analysis of DEGs. A functional enrichment analysis of DEGs, including gene ontology (GO) functional analysis and Kyoto Encyclopedia of Genes and Genomes (KEGG) pathway analysis, was performed using the Database for Annotation Visualization and Integrated
Discovery (DAVID; https://david.ncifcrf.gov/). GO analysis included the categories of cellular component (CC), biological process (BP), and molecular function ( $\mathrm{MF}) . \mathrm{P}<0.05$ was considered to indicate a statistically significant difference.

\section{Results}

Gene expression changes in tumors prior to and following treatment in drug-resistant patients with EGFR-activating BRAF mutations. A total of 6 samples of melanoma from 3 patients prior to and following treatment from the GSE50535 dataset were analyzed. The analysis resulted in the identification of 94 significant DEGs (62 upregulated and 32 downregulated genes), which were categorized using GO analysis in DAVID (Fig. 1). EGFR and EGFR-associated mutations were also analyzed (Fig. 2).

In the CC ontology analysis (Fig. 1A), the majority of the significantly enriched genes were associated with the terms plasma membrane (PM; 30 genes, $33.25 \%$ of all DEGs; $\mathrm{P}=0.002)$, $\mathrm{PM}$ part (21 genes, 22.58\%; $\mathrm{P}=0.002)$ and sarcolemma [4 genes, including biglycan $(B G N)$, collagen type VI $\alpha-3$ chain (COL6A3), COL6A2, and calcium voltage-gated channel subunit $\alpha-1 \mathrm{C}(C A C N A 1 C) ; 4.30 \%$ of all DEGs; $\mathrm{P}=0.003$ ].

In the $\mathrm{BP}$ ontology analysis (Fig. 1B), 9 genes [including protein tyrosine phosphatase, receptor type C (PTPRC), coronin 1A (COROIA), IKAROS family zinc finger 1 $(I K Z F 1)$, clusterin $(C L U)$, cluster of differentiation 4 (CD4), inositol polyphosphate-5-phosphatase D (INPP5D), complement clq C chain $(C 1 Q C), C D 74$, and major histocompatibility complex, class II, DR- $\alpha$ (HLA-DRA)] were enriched in the positive regulation of immune system process $\left(\mathrm{P}=1.08 \times 10^{-5}\right)$. Other categories with significant enrichment included immune response $(13 ; \mathrm{P}=0.003)$, positive regulation of lymphocyte activation $\left(6 ; \mathrm{P}=7.29 \times 10^{-5}\right)$, and lymphocyte differentiation $\left(6 ; \mathrm{P}=9.84 \times 10^{-5}\right)$. A total of 8 genes were associated with the positive regulation of cell differentiation $\left(\mathrm{P}=7.29 \times 10^{-5}\right)$.

In the MF ontology (Fig. 1C), categories with significant enrichment of DEGs included extracellular matrix structural constituent (5 genes: BGN, elastin, COL6A2, CD4, and COL5A1; $\left.\mathrm{P}=5.43 \times 10^{-4}\right)$, glycoprotein binding (4 genes; $\mathrm{P}=5.61 \times 10^{-4}$ ), kinase binding [six genes: PTPRC, COROIA, integrin subunit $\beta$-2 (ITGB2), CD4, troponin I3, cardiac type (TNNI3), and topoisomerase II- $\beta(T O P 2 B) ; \mathrm{P}=0.001]$, and protein kinase binding [five genes: PTPRC, ITGB2, CD4, TNNI3 and TOP2B; $\mathrm{P}=0.003]$.

KEGG pathway analysis of the 94 DEGs was also performed (Table I). The most significantly enriched KEGG pathway was cell adhesion molecules (CAMs; 7 DEGs; $\mathrm{P}=1.70 \times 10^{-4}$ ). A total of 5 DEGs were enriched in the antigen processing and presentation pathway.

Gene changes in tumor compared with non-tumor melanoma samples. The present study aimed to improve the understanding of the differences between tumor samples from melanoma patients with EGFR-activating $B R A F$ mutations and non-tumor samples from melanoma patients. Melanoma samples (SRR961663, SRR961665 and SRR961667) from 3 patients prior to treatment were compared with 1 non-tumor sample from the TCGA cutaneous melanoma database. A 


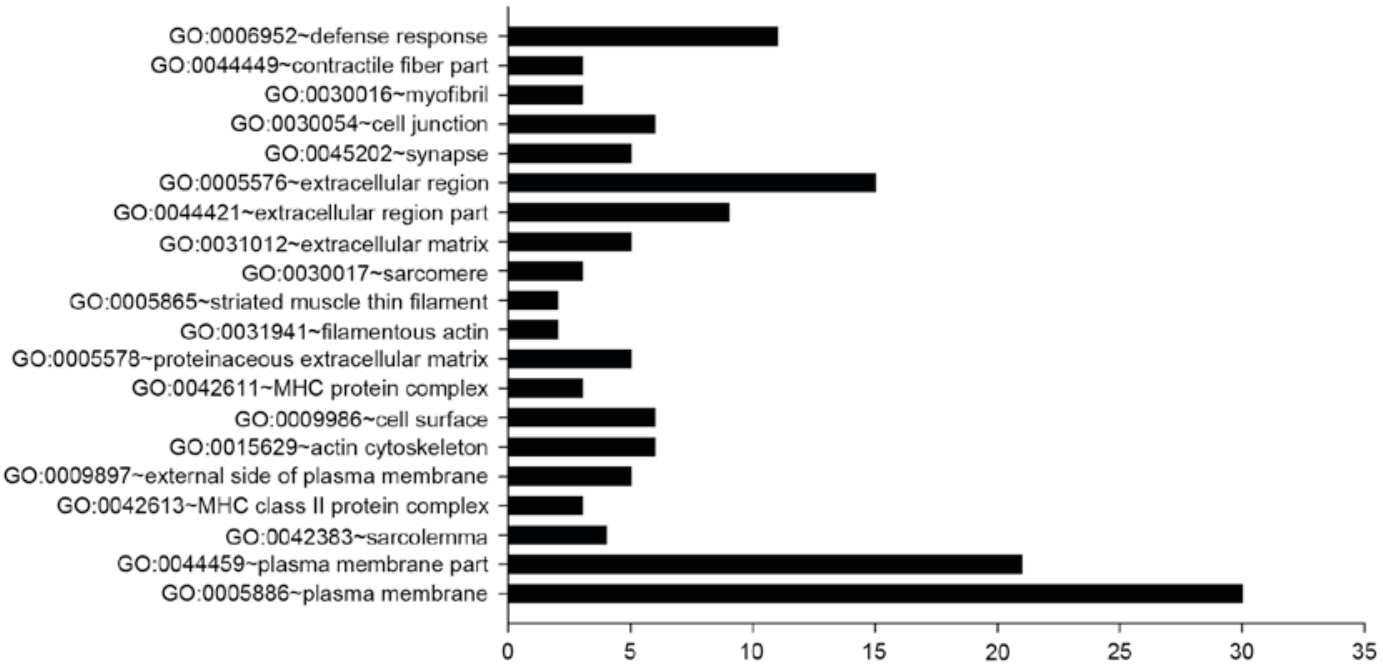

GO:0006952 defense response GO:0050870 positive regulation of $\mathrm{T}$ cell activation GO:0002252 immune effector process GO:0002521 leukocyte differentiation GO:0002449 -ymphocyte mediated immunity GO:0046649 lymphocyte activation GO:0006928 cell motion GO:0042110 T cell activation GO:0050730 regulation of peptidyl-tyrosine phosphorylation GO:0051094 positive regulation of developmental process GO:0030217 T cell differentiation GO:0050867 positive regulation of cell activation

GO:0019724 B cell mediated immunity GO:0002696 positive regulation of leukocyte activation GO:0016064 immunoglobulin mediated immune response GO:0030098 lymphocyte differentiation GO:0051251 positive regulation of lymphocyte activation GO:0045597 positive regulation of cell differentiation GO:0006955-immune response GO:0002684 positive regulation of immune system process

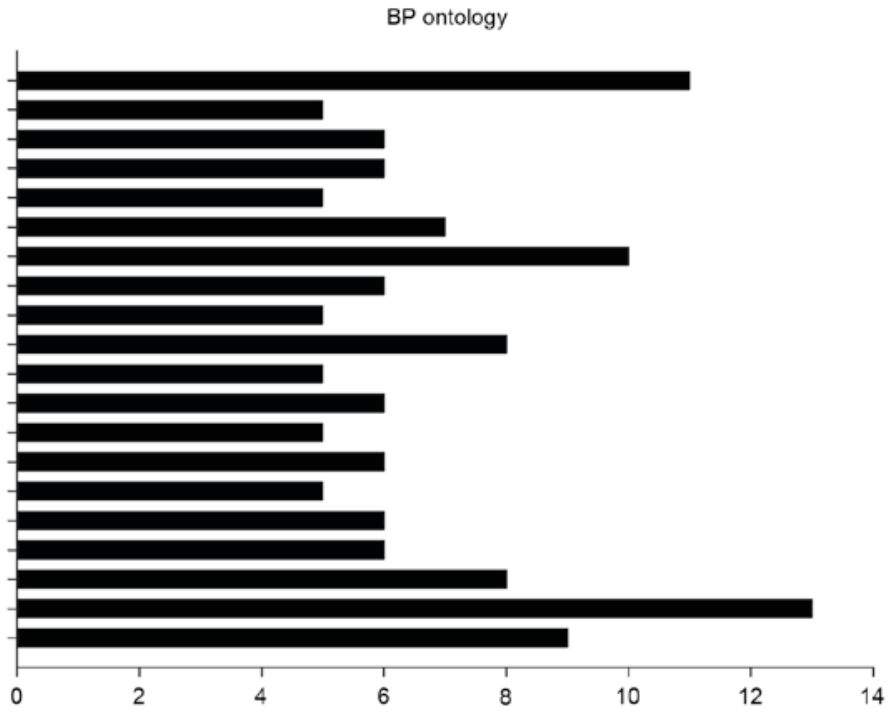

C

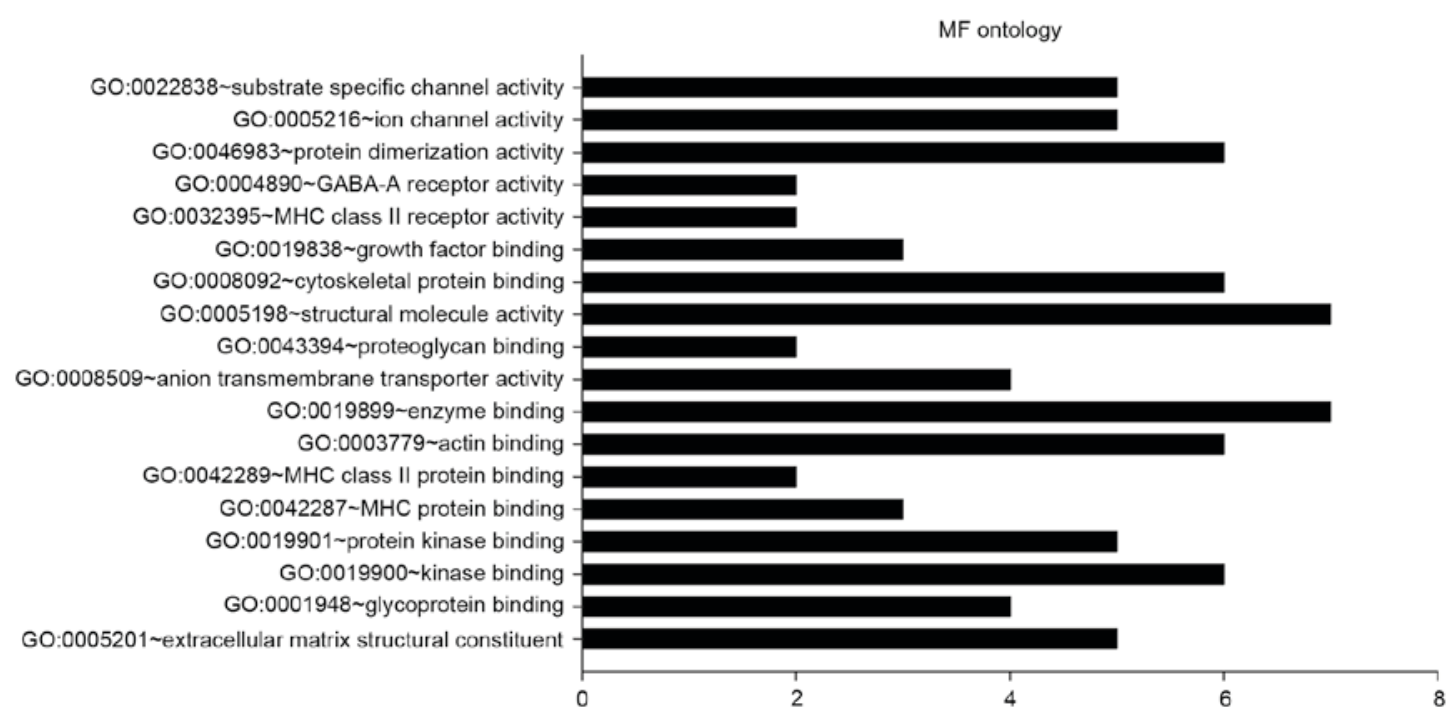

Figure 1. Differentially expressed genes between samples prior to and following treatment, with significant enrichment in (A) CC ontology, (B) BP and (C) MF ontology terms. CC, cellular component; BP, biological process; MF, molecular function. 
Table I. Top 5 Kyoto Encyclopedia of Genes and Genomes pathway terms with significant enrichment of DEGs in patients with epidermal growth factor receptor-activating BRAF mutations prior to and following treatment.

\begin{tabular}{lcc}
\hline Term & DEGs, $\mathrm{n}$ & P-value \\
\hline $\begin{array}{l}\text { HSA04514: Cell adhesion } \\
\text { molecules }\end{array}$ & 7 & $1.70 \times 10^{-4}$ \\
$\begin{array}{l}\text { HSA04612: Antigen processing } \\
\text { and presentation }\end{array}$ & 5 & $1.88 \times 10^{-3}$ \\
$\begin{array}{l}\text { HSA04940: Type I diabetes mellitus } \\
\text { HSA05414: Dilated cardiomyopathy }\end{array}$ & 4 & $2.41 \times 10^{-3}$ \\
$\begin{array}{l}\text { HSA04672: Intestinal immune } \\
\text { network for IgA production }\end{array}$ & 5 & $2.75 \times 10^{-3}$ \\
& & $3.75 \times 10^{-3}$ \\
\hline
\end{tabular}

DEG, differently expressed gene.

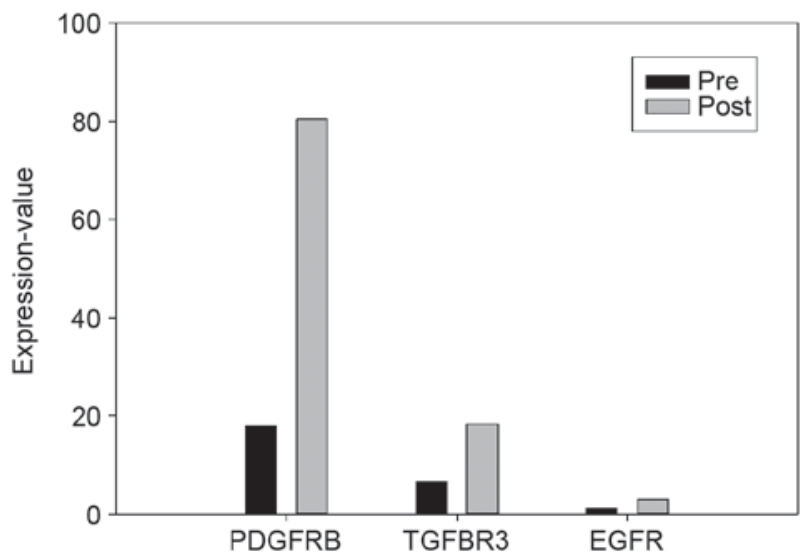

Figure 2. EGFR and EGFR-associated genes with altered expression levels prior to and following treatment for melanoma. EGFR, epidermal growth factor receptor; PDGFRB, platelet-derived growth factor receptor- $\beta$; TGFBR3, transforming growth factor- $\beta$ receptor 3; Pre, samples taken prior to melanoma treatment; Post, samples taken following melanoma treatment.

total of 274 significant DEGs were identified, which were then categorized with GO analysis in DAVID.

In the CC ontology analysis (Fig. 3A), the most significantly enriched categories were adhesion-associated items pertaining to the ribosome, including the cytosolic ribosome (27 genes; $\mathrm{P}=3.27 \times 10^{-28}$ ), ribosome ( 34 genes; $\left.\mathrm{P}=2.59 \times 10^{-24}\right)$, ribosomal subunit (26 genes; $\left.\mathrm{P}=2.59 \times 10^{-21}\right)$, cytosolic small ribosomal subunit (15 genes; $\left.\mathrm{P}=3.38 \times 10^{-13}\right)$, MHC protein complex (11 genes; $\mathrm{P}=1.03 \times 10^{-8}$ ) and MHC class I protein complex (6 genes; $\left.\mathrm{P}=5.25 \times 10^{-5}\right)$.

In the $\mathrm{BP}$ ontology analysis (Fig. 3B), the majority of categories with significant DEG enrichment were associated with the translation process, including translational elongation (33 genes; $\mathrm{P}=1.28 \times 10^{-35}$ ) and translation (37 genes; $\mathrm{P}=1.50 \times 10^{-22}$ ). DEGs were also enriched in immune-associated categories, including immune response ( 39 genes; $\mathrm{P}=1.08 \times 10^{-13}$ ), antigen processing and presentation ( 15 genes; $\left.\mathrm{P}=5.67 \times 10^{-12}\right)$, and antigen processing and presentation of peptide antigens ( 9 genes; $\left.\mathrm{P}=2.59 \times 10^{-9}\right)$.

In the MF ontology analysis (Fig. 3C), categories with significant enrichment in DEGs included ribosome
Table II. Top 5 Kyoto Encyclopedia of Genes and Genomes pathway terms with significant enrichment in DEGs between tumor samples from patients with EGFR-activating BRAF mutations and non-tumor samples.

\begin{tabular}{lcc}
\hline Term & DEGs, $\mathrm{n}$ & P-value \\
\hline HSA03010: Ribosome & 30 & $6.20 \times 10^{-27}$ \\
$\begin{array}{l}\text { HSA04612: Antigen processing } \\
\text { and presentation }\end{array}$ & 17 & $4.64 \times 10^{-11}$ \\
$\begin{array}{l}\text { HSA05322: Systemic lupus } \\
\text { erythematosus }\end{array}$ & 14 & $4.09 \times 10^{-7}$ \\
$\begin{array}{l}\text { HSA05330: Allograft rejection } \\
\text { HSA05332: Graft-versus-host }\end{array}$ & 9 & $1.33 \times 10^{-6}$ \\
disease & 9 & $2.54 \times 10^{-6}$ \\
\end{tabular}

DEG, differently expressed gene.

structural constituents ( 31 genes; $\mathrm{P}=4.67 \times 10^{-26}$ ), structural molecule activity (40 genes; $\mathrm{P}=1.30 \times 10^{-16}$ ), RNA binding (27 genes; $\left.\mathrm{P}=1.73 \times 10^{-6}\right)$, MHC class II receptor activity (6 genes; $\mathrm{P}=3.43 \times 10^{-6}$ ) and $\mathrm{MHC}$ class I receptor activity (5 genes; $\mathrm{P}=5.64 \times 10^{-5}$ ).

To develop an improved understanding of the function of genetic differences, pathway analysis was performed with the 274 DEGs (Table II). KEGG pathway terms with significantly enriched DEGs included the ribosome (30 genes; $\left.\mathrm{P}=6.20 \times 10^{-27}\right)$, antigen processing and presentation (17 genes; $\left.\mathrm{P}=4.64 \times 10^{-11}\right)$, and systemic lupus erythematosus (14 genes; $\left.\mathrm{P}=4.09 \times 10^{-7}\right)$.

DEGs between tumor and non-tumor samples from melanoma patients. A total of 9 genes $(C 1 Q C$, calcium-dependent secretion activator (CADPS), CD74, CLU, CORO1A, formin 1, HLA-DPA1, HLA-DRA, and lymphocyte-specific protein 1) were differentially expressed in the two DEG analyses described above (prior to vs. following treatment, and tumor vs. non-tumor). The greatest enrichments of DEGs were identified in the GO category immune process $\left(\mathrm{P}=1.21 \times 10^{-6}\right)$ and the KEGG pathway antigen processing and presentation (17 genes; $\mathrm{P}=7.81 \times 10^{-4}$ ).

DEGs between BRAF V600E mutation patients with and without EGFR activation. Finally, DEGs between samples taken from tumor and non-tumor sites of patients with the $B R A F$ V600E mutation of unknown EGFR activation status from the TCGA cutaneous melanoma database were analyzed. A total of 27 DGEs changed in the two groups. GO analysis with DAVID revealed that nine genes were enriched in the immune response category $\left(\mathrm{P}=1.22 \times 10^{-6}\right)$, and eight genes were enriched in the defense response category $\left(\mathrm{P}=7.96 \times 10^{-6}\right)$.

\section{Discussion}

Gene expression alterations in the tumors of patients with EGFR-activating BRAF V600E mutations prior to and following BRAF inhibitor treatment were analyzed. Samples taken from tumor and non-tumor sites in patients with BRAF V600E mutations of unknown EGFR activation status were 

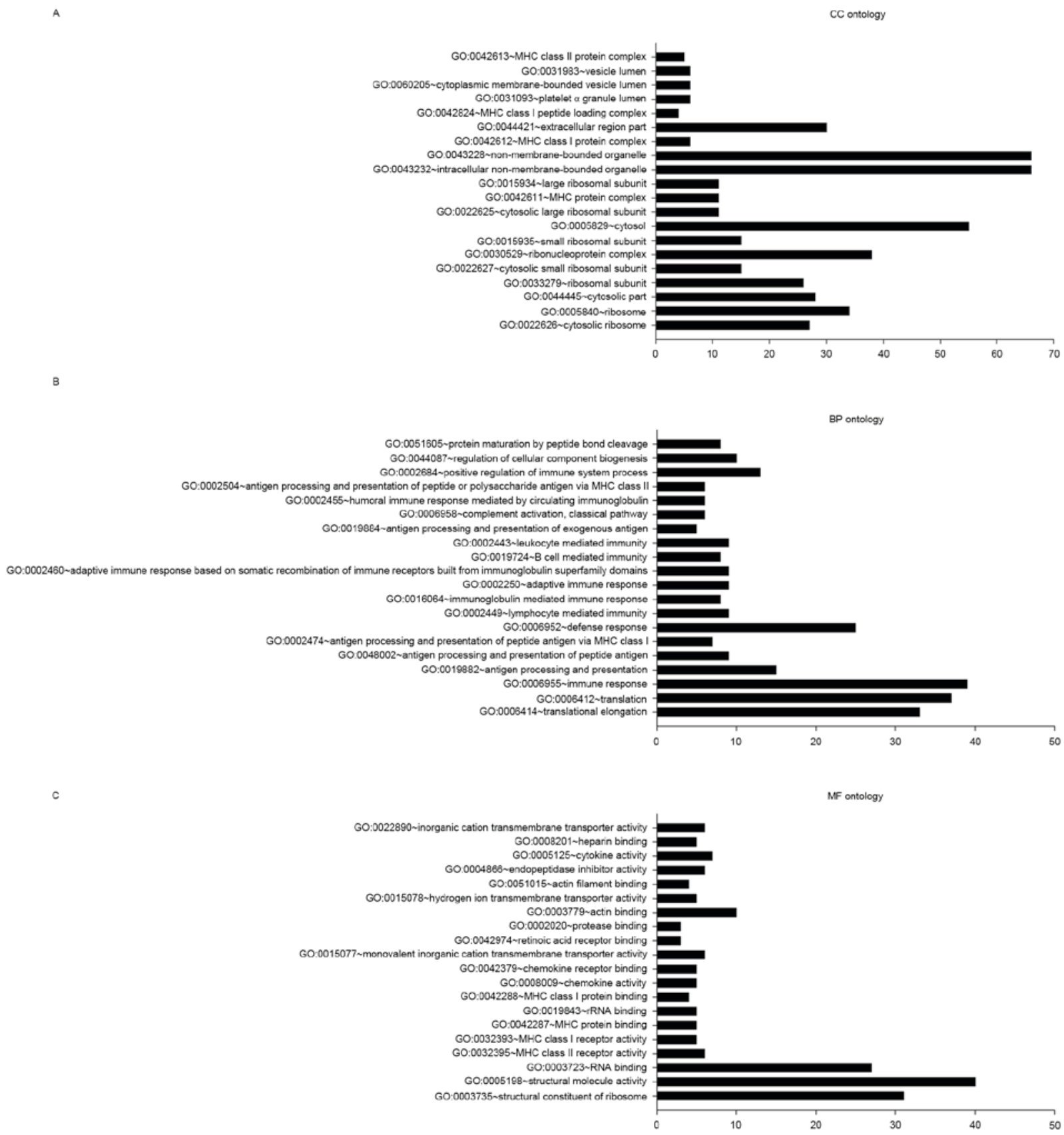

Figure 3. Differentially expressed genes between samples from epidermal growth factor receptor-expressing tumors with BRAF mutations and non-cancer tissue with significant enrichment in (A) CC, (B) BP and (C) MF ontology terms. CC, cellular component; BP, biological process; MF, molecular function.

also compared. The functions of DEGs were analyzed by GO annotation and KEGG pathway enrichment analyses. The study aimed to provide information to guide the development of novel therapeutic strategies for melanoma patients with EGFR activation who are resistant to typical drugs.

RNA-sequencing (RNA-seq) technology is a powerful tool for analyzing gene expression. Using RNA-seq data for melanoma patients with EGFR activation, 94 genes were identified that were differentially expressed in samples prior to and following treatment, including 62 upregulated and 32 downregulated genes. Gene functional annotation revealed 30 genes associated with membranes in CC oncology, which provides a possible direction for future studies.
Ion channels on the membrane are involved in numerous tumor cell activities, including cell proliferation, differentiation, secretion and survival $(19,20)$. A significant upregulation of CACNAIC ( $\left.\mathrm{P}=5.00 \times 10^{-5}\right)$, which encodes the $\alpha-1$ subunit of a voltage-dependent calcium channel located on the PM (21), was observed; this gene is not detectable in normal tissues (22). Calcium channel proteins have been associated with primary tumors of the colon, lung and skin (23). Certain drugs targeting CACNA1C, including magnesium sulfate and nicardipine, have been reported; magnesium sulfate can be used to inhibit the action potential of muscle cells, thereby reducing the frequency and strength of contractions (24); nicardipine is a potent calcium channel inhibitor with important 
vasodilatory and antihypertensive characteristics that can be used to enhance the efficacy of certain antitumor agents (25).

A significant downregulation of solute carrier family 4 member $10(S L C 4 A 10)\left(\mathrm{P}=5.00 \times 10^{-5}\right)$, which belongs to a small family of sodium-coupled bicarbonate transporters that regulate the intracellular $\mathrm{pH}$ of neurons (25), was also observed. In addition to unlimited cell proliferation, cancer is characterized by an altered cellular environment that promotes tumor cell proliferation and metastasis (26). PH homeostasis in any cell type is a complicated process. In tumor cells, these processes are even more complex owing to the internal compartment being slightly more alkaline ( $\mathrm{pH} 7.4$ or more) and the external compartment being more acidic than in normal cells (27). Downregulation of $S L C 4 A 10$, leading to decreased $\mathrm{Cl}^{-} / \mathrm{HCO}_{3}$ transport, may be associated with environmental alterations for melanoma growth.

A total of 274 genes were identified that were differentially expressed between untreated EGFR-activated melanoma samples and non-tumor samples from the TCGA Database. GO and pathway analyses revealed that numerous genes involved in immune-associated processes, particularly antigen processing and presentation processes, were enriched. One enriched gene was $C D 74$, which encodes a protein associated with the class II major histocompatibility complex (MHC) and is a chaperone that regulates antigen presentation. CD74 serves as a cell-surface receptor for the cytokine macrophage migration inhibitory factor, which, when bound to the encoded protein, initiates survival pathways and cell proliferation. A previous study indicated that CD74 is only expressed in melanoma cells and not in benign melanocytes (28). Milatuzumab is a drug used for the treatment of tumors expressing the CD74 antigen (29).

Antigen presentation serves a key role in the development of melanoma vaccines (30). Broadly speaking, a tumor cell is also an antigen-presenting cell. Tumor cells form a complex with MHC class I molecules via the cytosolic processing pathway, with tumor antigens on the cell surface being recognized by $\mathrm{CD}^{+} \mathrm{T}$ cells. Alternatively, tumor cells can form MHC class I or II molecules by lysosomal processing of tumor antigens from dendritic cells or specialized antigen-presenting macrophages. These tumor cells are then recognized by $\mathrm{CD}^{+} / \mathrm{CD}^{+} \mathrm{T}$ cells $(30)$.

Significant enrichment was observed in numerous genes associated with ribosomal processes. Ribosome synthesis and translational control are essential processes for cells. Several tumor suppressor genes and proto-oncogenes can affect the formation or modification of ribosomes (31). However, the mechanisms by which these genes affect ribosomes remain unclear at present and further experiments are required.

The present study analyzed tumor and non-tumor samples from patients with BRAF V600E mutations of unknown EGFR status from the TCGA Database. These results were compared with DEGs between tumor samples from patients with EGFR-activating BRAF V600E mutations prior to and following treatment. The expression of 27 genes was altered in both comparisons. These genes were predominantly enriched in categories associated with immune response, which suggests that using immunotherapy in the early stages of melanoma may be a valid therapeutic approach. The expression of 9 genes was altered when comparing tumor and non-tumor tissues, and treated and untreated tumors. The majority of these genes exhibited different changes at different stages: For example, a gene that was downregulated in the tumor area compared with the non-tumor area might be upregulated upon acquiring resistance following treatment. DEGs that were significantly enriched in the immune process category by GO analysis included $C L U, C 1 Q C, C D 74$, and $H L A-D R A$. The C1QC protein is the target of several drugs, as it is a component of the human complement system. Studies have associated a lack of C1QA with lupus and glomerulonephritis (32). Several C1QC-targeting drugs, including tositumomab, palivizumab and cetuxima, are used clinically to treat cancer (22). CLU has been shown to be associated with a number of biological processes, including apoptosis and tumor development, as well as neurodegenerative diseases.

The present study has several deficiencies. The sample size was relatively small, with only 3 samples with EGFR activation and 1 non-tumor sample; the small sample size may lead inaccuracies when comparing the tumor and non-tumor samples. In order to improve the accuracy of the results of the present study, the authors will continue to collect samples with the relevant mutations for further research.

In conclusion, the treatment of melanoma is a complex process. Significant changes were observed in genes associated with the PM of $B R A F$ inhibitor-resistant melanoma patients with EGFR-activated tumors prior to and following treatment. Significant changes in immune process-associated genes were also identified in melanoma patients between tumor and non-tumor samples. Although these findings may provide direction for clinical melanoma-specific therapy, follow-up studies on melanoma are required.

\section{Acknowledgements}

The present study was funded by grants from the Natural Science Foundation of China (grant nos.81371719), the Colleges Pearl River Scholar Funded Scheme (grant no., GDUPS, 2013) and Guangdong Natural Science Foundation (grant nos. 2014A030312013).

\section{References}

1. Chapman PB, Hauschild A, Robert C, Haanen JB, Ascierto P, Larkin J, Dummer R, Garbe C, Testori A, Maio M, et al: Improved survival with vemurafenib in melanoma with BRAF V600E mutation. N Engl J Med 364: 2507-2516, 2011.

2. Clary BM, Brady MS, Lewis JJ and Coit DG: Sentinel lymph node biopsy in the management of patients with primary cutaneous melanoma: Review of a large single-institutional experience with an emphasis on recurrence. Ann Surg 233: 250-258, 2001.

3. Davies H, Bignell GR, Cox C, Stephens P, Edkins S, Clegg S, Teague J, Woffendin H, Garnett MJ, Bottomley W, et al: Mutations of the BRAF gene in human cancer. Nature 417: 949-954, 2002.

4. Flaherty KT, Hodi FS and Fisher DE: From genes to drugs: Targeted strategies for melanoma. Nat Rev Cancer 12: 349-361, 2012.

5. Hartsough EJ, Basile KJ and Aplin AE: Beneficial effects of RAF inhibitor in mutant BRAF splice variant-expressing melanoma. Mol Cancer Res 12: 795-802, 2014.

6. Heidorn SJ, Milagre C, Whittaker S, Nourry A, Niculescu-Duvas I, Dhomen N, Hussain J, Reis-Filho JS, Springer CJ, Pritchard C and Marais R: Kinase-dead BRAF and oncogenic RAS cooperate to drive tumor progression through CRAF. Cell 140: 209-221, 2010.

7. Vial E, Sahai E and Marshall CJ: ERK-MAPK signaling coordinately regulates activity of Racl and RhoA for tumor cell motility. Cancer Cell 4: 67-79, 2003. 
8. Gautschi O, Pauli C, Strobel K, Hirschmann A, Printzen G, Aebi S and Diebold J: A patient with BRAF V600E lung adenocarcinoma responding to vemurafenib. J Thorac Oncol 7 : e23-e24, 2012

9. Das Thakur M, Salangsang F, Landman AS, Sellers WR Pryer NK, Levesque MP, Dummer R, McMahon M and Stuart DD: Modelling vemurafenib resistance in melanoma reveals a strategy to forestall drug resistance. Nature 494 $251-255,2013$

10. Kalluri R and Zeisberg M: Fibroblasts in cancer. Nat Rev Cancer 6: 392-401, 2006

11. McMillin DW, Delmore J, Weisberg E, Negri JM, Geer DC Klippel S, Mitsiades N, Schlossman RL, Munshi NC, Kung AL, et al: Tumor cell-specific bioluminescence platform to identify stroma-induced changes to anticancer drug activity. Nat Med 16: 483-489, 2010.

12. Lovly CM, Dahlman KB, Fohn LE, Su Z, Dias-Santagata D, Hicks DJ, Hucks D, Berry E, Terry C, Duke M, et al: Routine multiplex mutational profiling of melanomas enables enrollment in genotype-driven therapeutic trials. PLoS One 7: e35309, 2012

13. Chaft JE, Oxnard GR, Sima CS, Kris MG, Miller VA and Riely GJ: Disease flare after tyrosine kinase inhibitor discontinuation in patients with EGFR-mutant lung cancer and acquired resistance to erlotinib or gefitinib: Implications for clinical trial design. Clin Cancer Res 17: 6298-6303, 2011.

14. Corcoran RB, Ebi H, Turke AB, Coffee EM, Nishino M, Cogdill AP, Brown RD, Della Pelle P, Dias-Santagata D, Hung KE, et al: EGFR-mediated re-activation of MAPK signaling contributes to insensitivity of BRAF mutant colorectal cancers to RAF inhibition with vemurafenib. Cancer Discov 2 : 227-235, 2012.

15. Prahallad A, Sun C, Huang S, Di Nicolantonio F, Salazar R, Zecchin D, Beijersbergen RL, Bardelli A and Bernards R: Unresponsiveness of colon cancer to BRAF(V600E) inhibition through feedback activation of EGFR. Nature 483: 100-103, 2012

16. Sun C, Wang L, Huang S, Heynen GJ, Prahallad A, Robert C, Haanen J, Blank C, Wesseling J, Willems SM, et al: Reversible and adaptive resistance to BRAF(V600E) inhibition in melanoma. Nature 508: 118-122, 2014.

17. Trapnell C, Roberts A, Goff L, Pertea G, Kim D, Kelley DR, Pimentel H, Salzberg SL, Rinn JL and Pachter L: Differential gene and transcript expression analysis of RNA-seq experiments with TopHat and Cufflinks. Nat Protoc 7: 562-578, 2012.

18. Robinson MD, McCarthy DJ and Smyth GK: edgeR: A Bioconductor package for differential expression analysis of digital gene expression data. Bioinformatics 26: 139-140, 2010.

19. Arcangeli A, Crociani O, Lastraioli E, Masi A, Pillozzi S and Becchetti A: Targeting ion channels in cancer: A novel frontier in antineoplastic therapy. Curr Med Chem 16: 66-93, 2009.
20. Cheli VT, Santiago González DA, Spreuer V and Paez PM: Voltage-gated $\mathrm{Ca} 2+$ entry promotes oligodendrocyte progenitor cell maturation and myelination in vitro. Exp Neurol 265: 69-83, 2015.

21. Tsavaler L, Shapero MH, Morkowski S and Laus R: Trp-p8, a novel prostate-specific gene, is up-regulated in prostate cancer and other malignancies and shares high homology with transient receptor potential calcium channel proteins. Cancer Res 61: 3760-3769, 2001

22. Imming $P$, Sinning $C$ and Meyer A: Drugs, their targets and the nature and number of drug targets. Nat Rev Drug Discov 5: 821-834, 2006.

23. Kunzelmann K: Ion channels and cancer. J Membr Biol 205 : 159-173, 2005.

24. Chen X, Ji ZL and Chen YZ: TTD: Therapeutic target database. Nucleic Acids Res 30: 412-415, 2002.

25. Gurnett CA, Veile R, Zempel J, Blackburn L, Lovett M and Bowcock A: Disruption of sodium bicarbonate transporter SLC4A10 in a patient with complex partial epilepsy and mental retardation. Arch Neurol 65: 550-553, 2008.

26. Hanahan D and Weinberg RA: Hallmarks of cancer: The next generation. Cell 144: 646-674, 2011

27. Neri D and Supuran CT: Interfering with $\mathrm{pH}$ regulation in tumours as a therapeutic strategy. Nat Rev Drug Discov 10: 767-777, 2011.

28. Ong GL, Goldenberg DM, Hansen HJ and Mattes MJ: Cell surface expression and metabolism of major histocompatibility complex class II invariant chain (CD74) by diverse cell lines. Immunology 98: 296-302, 1999.

29. Kaufman JL, Niesvizky R, Stadtmauer EA, Chanan-Khan A, Siegel D, Horne H, Wegener WA and Goldenberg DM: Phase I, multicentre, dose-escalation trial of monotherapy with milatuzumab (humanized anti-CD74 monoclonal antibody) in relapsed or refractory multiple myeloma. Br J Haematol 163: 478-486, 2013.

30. Thompson JA, Srivastava MK, Bosch JJ, Clements VK, Ksander BR and Ostrand-Rosenberg S: The absence of invariant chain in MHC II cancer vaccines enhances the activation of tumor-reactive type $1 \mathrm{CD} 4+\mathrm{T}$ lymphocytes. Cancer Immunol Immunother 57: 389-398, 2008.

31. Ruggero D and Pandolfi PP: Does the ribosome translate cancer? Nat Rev Cancer 3: 179-192, 2003.

32. Weening JJ, D'Agati VD, Schwartz MM, Seshan SV, Alpers CE, Appel GB, Balow JE, Bruijn JA, Cook T, Ferrario F, et al: The classification of glomerulonephritis in systemic lupus erythematosus revisited. Kidney Int 65: 521-530, 2004. 